\title{
Descripción micro-anatómica de las larvas de Engraulis anchoita y su aplicación en estudios de condición nutricional
}

Micro-anatomical description of Engraulis anchoita larvae and its employment to diagnose nutritional condition

\author{
Marina V. Diaz ${ }^{1,2}$, H. Ernesto Christiansen², Marcelo Pájaro² y Gustavo J. Macchi ${ }^{1,2}$ \\ ${ }^{1}$ Consejo Nacional de Investigaciones Científicas y Técnicas (CONICET), Rivadavia 1917, C1033AAV, Ciudad Autónoma \\ de Buenos Aires, Argentina \\ ${ }^{2}$ Instituto Nacional de Investigación y Desarrollo Pesquero, INIDEP, Paseo Victoria Ocampo 1, Escollera Norte, B7602HSA, \\ Mar del Plata, Argentina.mdiaz@inidep.edu.ar
}

\begin{abstract}
Diverse indices have been used to characterize the nutritional condition of fish larvae. Histological criteria were shown to be reliable in diagnosing nutritional condition, because they involve the study of cellular aspect, structure and tissular integrity. However, since the microscopical appearance of larval tissues varies both as a consequence of nutritional status and developmental stages, it becomes necessary to characterize the employed structures and to take into account the developmental stage of the studied specimen. The aim of this study was to develop a typical micro-anatomical description to assess the nutritional condition of Engraulis anchoita fish larvae collected in the Argentine Sea. Several tissues were diagnosed to calculate a histological condition index $(\mathrm{ICH})$ in regards to anchovy larvae ontogeny. In addition, the tissues analysed were divided into short and long-term indices. A reduction of $\mathrm{HCl}$ was observed during yolk exhaustion and at the end of the larval stage. In order to detect slight changes in histological condition, short term tissues proved to be more sensitive to starvation than long term tissues.
\end{abstract}

Key words: Engraulidae, southwest Atlantic Ocean, histology, starvation

\begin{abstract}
Resumen.- Diversos índices han sido empleados para caracterizar la condición nutricional en larvas de peces. Los criterios histológicos han demostrado ser confiables para diagnosticar la condición ya que involucran el estudio del aspecto de las células y la estructura e integridad de diferentes tejidos de las larvas. Sin embargo, debido a que el aspecto microscópico de los tejidos varía tanto como consecuencia de la condición nutricional como del estadio de desarrollo de las larvas, se hace necesario caracterizar las estructuras empleadas para diagnosticar su condición nutricional y tener en cuenta el estadio de desarrollo de los ejemplares. El objetivo principal de este trabajo fue una descripción micro-anatómica de los tejidos utilizados para determinar la condición nutricional de larvas de peces con larvas de Engraulis anchoita recolectadas en el mar Argentino. Se determinó un índice de condición histológica $(\mathrm{ICH})$ para diferentes estadios de desarrollo de larvas de anchoíta, discriminándose entre los tejidos que muestran inanición sufrida a largo plazo y los que muestran inanición a corto plazo. Se observó una reducción en el $\mathrm{ICH}$ luego de la reabsorción del vitelo y hacia el final de la etapa larval. Los tejidos de corto plazo mostraron mayor sensibilidad a pequeños cambios en la condición nutricional.
\end{abstract}

Palabras clave: Engraulidae, Océano Atlántico sudoccidental, histología, inanición

\section{INTRODUCCIÓN}

En los teleósteos marinos que desovan huevos pelágicos, la mayoría de los sistemas de órganos se originan durante el periodo embrionario tardío o durante la etapa larval vitelina y se hacen funcionales hacia el final de esta etapa. El periodo de larva vitelina dura de unos pocos días a una semana dependiendo principalmente de la temperatura, y una vez que se ha agotado el vitelo, en general las larvas deben comenzar a alimentarse rápidamente (Jobling 1995,
Fuiman 2002). A lo largo del desarrollo ontogénico, los requerimientos nutricios de las larvas se incrementan constantemente y los hábitos alimenticios, los patrones de locomoción y otras características se transforman. La maduración de los sistemas de órganos debe involucrar los cambios funcionales relacionados con modificaciones en la ecología de las larvas (O’Connell 1981). 
Es ampliamente aceptado que la inanición es una de las causas principales de mortalidad larval. El estudio del estado nutricional permite evaluar individualmente el estado fisiológico de las larvas que es reflejo de las condiciones ambientales a las que han sido expuestas, permitiendo así la estimación de la incidencia de la mortalidad embrionaria. Las técnicas histológicas permiten estudiar la condición nutricional a través de las modificaciones en la apariencia de las células y sus arreglos en diferentes tejidos del animal. Esto es posible ya que la histología de las larvas privadas de alimento es diferente a la de los animales bien alimentados.

La temperatura determina el metabolismo larval, las tasas de crecimiento y el desarrollo de los órganos, así como también la relación entre el estadio de desarrollo y la longitud de los ejemplares (Jobling 1995). De este modo, cuando se investiga la inanición en distintos ambientes, los resultados deberían ser interpretados considerando tanto las características ambientales como los estadios de desarrollo de las larvas (Sieg 1992). Debido a que los estudios histológicos excluyen los análisis de edad empleando los otolitos, Sieg (1998) propone emplear un método alternativo para clasificar estadios de desarrollo larval para Engraulis anchoita Hubbs \& Marini, 1935. Este método requiere el estudio histológico de ciertos tejidos de cada larva (Tabla 1).

Engraulis anchoita se distribuye tanto en el área costera como de plataforma en un amplio rango latitudinal $\left(23-47^{\circ} \mathrm{S}\right)$ desde Cabo Frío, Brasil, hasta el extremo sur del Golfo San Jorge, Argentina (Sánchez 1995). Existen al menos dos poblaciones, la población bonaerense al norte de los $41^{\circ} \mathrm{S}$ y la patagónica entre $41^{\circ}$ y $47^{\circ} \mathrm{S}$ las que se reproducen durante primavera y verano austral, respectivamente (Brandhorst et al. 1974). La población norte o bonaerense de anchoíta, objeto de estudio en el presente trabajo, conforma el grupo de peces pelágicos más importante de la región, tanto por su biomasa (Ciechomski \& Sánchez 1988) como por su importancia en la trama trófica. Si bien constituye un recurso poco explotado en la actualidad, posee una gran potencialidad pesquera, y debido a la situación pesquera mundial y local, E. anchoita podría ser explotada más intensamente en el futuro, por lo cual se hace necesario profundizar los estudios sobre su biología. En particular, debido a la elevada mortalidad embrionaria y larval de esta especie (Sánchez 1995), los estudios sobre los primeros estadios de desarrollo son de gran importancia.

La morfología externa de las larvas de anchoíta a lo largo del desarrollo ontogénico ha sido descrita por
Tabla 1. Criterio histológico para clasificar los estadios de desarrollo de las larvas de Engraulis anchoita, tomado de Sieg (1998). ED: estadio de desarrollo / Histological criteria used to classify different organ developmental stages of Engraulis anchoita larvae, taken from Sieg (1998). ED: developmental stage

ED I Larvas vitelinas. Se detecta claramente una masa vitelina o remanentes en posición anterior al hígado en desarrollo.

ED II Sin vitelo detectable. Tubo digestivo recto, quijada ventral con una capa simple de condrocitos, primer esbozo de vejiga gaseosa.

ED III Tubo digestivo todavía recto. La altura del epitelio del esófago comienza a incrementarse. El páncreas se extiende hacia la zona craneal. La quijada ventral está formada por más de una capa de condrocitos. La vejiga gaseosa aumenta de tamaño.

ED IV Mucosa del tubo digestivo medio plegada en la parte ventral, la parte dorsal comienza a plegarse, el tubo digestivo posterior sin plegamientos. La vejiga gaseosa continúa incrementándose en tamaño y puede formar una pequeña cavidad.

ED V Mucosa del tubo digestivo medio plegada dorsal y centralmente, el tubo digestivo posterior comienza a plegarse. Vejiga gaseosa con varias capas de células.

ED VI Tubo digestivo posterior completamente plegado. La altura del epitelio del esófago se incrementa de manera importante, con diferentes capas celulares. El páncreas se extiende craneal y caudalmente. La vejiga gaseosa presenta una pared delgada debido al primer llenado, puede mostrase muy extendida.

Ciechomski (1965) y Phonlor (1984). Sieg (1995) realizó una descripción histológica e histoquímica de los estadios larvales de esta especie; sin embargo, estos resultados no han sido publicados hasta el momento.

Debido a que el aspecto microscópico de los tejidos varía tanto como consecuencia de la condición nutricional como del estadio de desarrollo de los individuos, se hace necesario caracterizar las estructuras empleadas para diagnosticar su condición nutricional y tener en cuenta el estadio de desarrollo de los ejemplares. Los tejidos generalmente empleados son: el tubo digestivo, hígado, músculo, cerebro u otros tejidos que muestren variaciones en función del tratamiento alimenticio para la especie de estudio. Por otra parte, de acuerdo a la literatura disponible, 
los distintos tejidos responden de manera diferente a la inanición (Theilacker 1978, Watanabe 1985, Oozeki et al. 1989, McFadzen et al. 1994). Por lo tanto en este estudio se han clasificado como tejidos de respuesta a la inanición a largo plazo al tejido nervioso, cartílago, músculo y notocorda, y como de respuesta corto plazo al tubo digestivo, páncreas e hígado.

El objetivo principal de este trabajo fue la descripción micro-anatómica de siete tejidos utilizados para determinar la condición nutricional de larvas de peces empleando larvas de Engraulis anchoita recolectadas en el mar Argentino. Se determinó un índice de condición histológica $(\mathrm{ICH})$ para diferentes estadios de desarrollo de larvas de anchoíta, discriminándose entre los tejidos que muestran inanición sufrida a largo plazo y los que muestran inanición a corto plazo.

\section{Materiales Y MÉTOdoS}

Las larvas empleadas en este trabajo provienen de la campaña de Evaluación de la anchoíta Argentina realizada por el Instituto Nacional de Investigación y Desarrollo Pesquero (INIDEP) para la estimación de la abundancia de la población bonaerense de la especie (entre $34^{\circ}$ y $41^{\circ} \mathrm{S}$ ) durante el período reproductivo de 2006. Las muestras fueron recolectadas realizando lances verticales empleando una red PairoVET con un diámetro de boca de $25 \mathrm{~cm}$ y provista de una malla de $200 \mu \mathrm{m}$. Las larvas fueron separadas in situ, fijadas en formol buffer durante $2 \mathrm{~h}$ y posteriormente conservadas en alcohol $70 \%(\mathrm{~N}=191)$. Se registró la longitud estándar (LE) de cada una de ellas (distancia entre la punta del hocico y punta de la notocorda en larvas en preflexión notocordal, y distancia entre la punta del hocico y la base de los radios hipurales en larvas en postflexión notocordal) empleando una lupa Wild M8 con ocular graduado. Luego las larvas fueron deshidratadas en diluciones crecientes de etanol, aclaradas en xilol y finalmente incluidas en paraplast ${ }^{\circledR}$. Posteriormente se procedió a realizar cortes histológicos longitudinales de $4 \mu \mathrm{m}$ de espesor en forma seriada y se realizó una tinción con hematoxilina-eosina (HE). A cada ejemplar se le asignó un estadio de desarrollo de acuerdo a los estadios propuestos por Sieg (1998) que se presentan en la Tabla 1.

Por otra parte, empleando microscopía óptica (resolución de $0,1 \mu \mathrm{m}$ ), se realizó una descripción microscópica de los tejidos que usualmente son empleados para la caracterización histológica: tejido nervioso, cartílago, músculo, notocorda, tubo digestivo, páncreas e hígado. Finalmente, se realizó un diagnóstico histológico cualitativo de todos tejidos de cada una de las larvas para determinar su estado nutricional. Se le asignó un nivel de condición a cada tejido y luego se promediaron los valores obtenidos para todos los tejidos. De este modo, cada individuo fue caracterizado con un índice de condición histológica semi-cuantitativo (ICH) de 1 a 3 indicando inanición o buena condición, respectivamente. Como se mencionó previamente, debido a que los tejidos responden de diferente manera frente a la inanición, se calcularon separadamente los ICH a corto y largo plazo.

Los valores medios de longitud larval e ICH obtenidos para cada ED (= estadio de desarrollo) fueron comparados mediante un ANOVA de una vía seguido de un test de Tukey cuando se encontraron diferencias significativas, utilizando el software estadístico INFOSTAT®.

Para evitar factores subjetivos, los diagnósticos histológicos fueron realizados a modo ciego, sin tener información previa sobre la procedencia de la larva estudiada.

\section{Resultados}

Las larvas de Engraulis anchoita mostraron diferencias significativas en sus longitudes promedio entre los estadios de desarrollo $\left(\mathrm{F}_{(5,185)}=164,51 ; P<0,0001\right.$, Fig. 1). Sólo los estadios I y II mostraron similares longitudes corporales, mientras el resto de los estadios (III al VI) fueron todos distintos entre sí.

\section{DESCRIPCIÓN hiSTOLÓgICA PARA DIAgNOSIS A LARGo PLAZO}

En la descripción microscópica general de los tejidos de las larvas de Engraulis anchoita empleados para realizar diagnósticos histológicos, se individualizó la variabilidad en la micro-anatomía que muestran los ejemplares capturados en el ambiente natural.

Encéfalo (Fig. 2a-c): Para caracterizar la condición histológica del tejido nervioso se observó el aspecto de los cuerpos celulares del cerebro, el grado de asociación entre ellos y la presencia de espacios intercelulares. El tejido nervioso cerebral de las larvas en buena condición se observó en estado de normalidad en su parte glial y neuronal, bien irrigado, compacto e íntegro, los cuerpos celulares se presentaron asociados (Fig. 2a). Las larvas que mostraron una condición pobre presentaron una masa encefálica necrosada con neuropilo al parecer edematizado (Fig. 2b, c). 


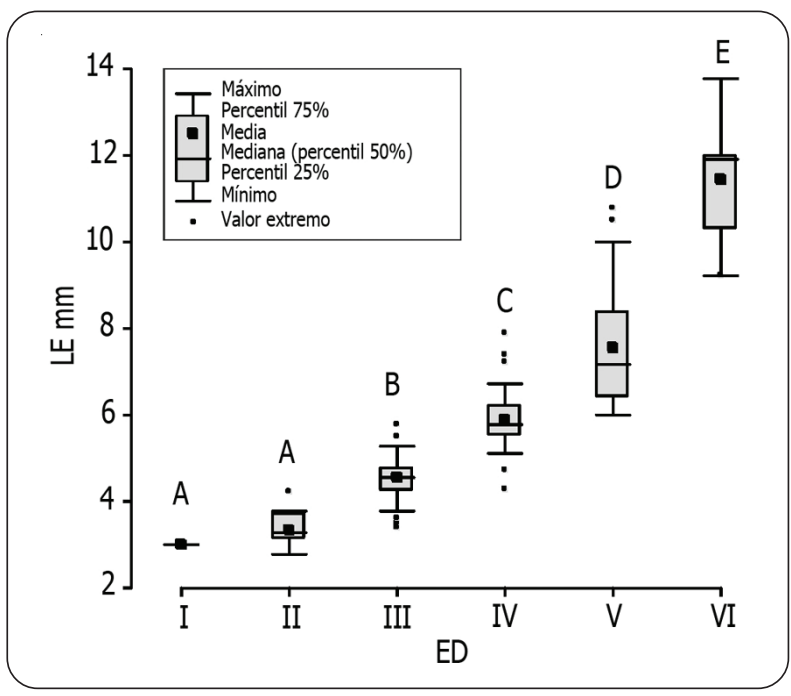

Figura 1. Diagrama de cajas (Box-plot) de la longitud estándar (LE) (mm) para los diferentes estadios de desarrollo (ED) de las larvas de Engraulis anchoita. Las letras distintas en mayúsculas indican diferencias significativas en las comparaciones según el test $a$ posteriori de Tukey $(P<0,001) /$ Box-plot diagram of standard length (LE) $(\mathrm{mm})$ obtained for each developmental stage (ED) of Engraulis anchoita. Different capital letters indicate significant differences $(P<0.001)$ among developmental stages, according to a posteriori Tukey's test

Cartílago (Fig. 2d-f): Las quijadas y los elementos branquiales, junto con ciertos elementos de la cintura pectoral y el cráneo, son tipificables como procartílago, compuestos por condrocitos muy próximos, separados por una delgada capa de matriz endocondral. Durante la etapa de procartílago, los condrocitos basófilos tienen núcleo prominente, redondeado, claro y granular (Fig. 2d). En las larvas que mostraron una buena condición también se observó el citoplasma levemente teñido llenando completamente los espacios capsulares. En larvas que mostraron estadios necrobióticos incipientes o avanzados, el núcleo se caracterizó por ser pequeño y denso y el citoplasma pudo faltar total o parcialmente como neta manifestación de muerte celular (Fig. 2e, f).

Musculatura del tronco (Fig. 2g-i): El endomisio de la musculatura del tronco está estrechamente insertado a las superficies laterales del conjuntivo de la notocorda, entre la médula espinal y la aorta dorsal. La musculatura está segmentada en miómeros en forma de «V»y solapándose levemente; la capa es delgada durante el comienzo del desarrollo.

Los principales componentes de la musculatura del tronco son una capa de miocélulas internas oblicuas, antero-ventral a postero-dorsal, y una capa externa de miocélulas oblicuas de alineación opuesta. Las fibras musculares contienen un número de núcleos axiales rodeados por miofibrillas que corren a lo largo de toda la longitud de la fibra con evidentes estriaciones, están ampliamente unidas a los mioseptos y precisamente alineadas en miómeros sucesivos. El tronco muscular en las larvas en buena condición se observó compacto, con las fibras musculares paralelas y asociadas (Fig. 2g). Se observaron núcleos prominentes y nucleolos distinguibles. En las larvas en mala condición las fibras musculares se observaron delgadas, retraídas, separadas, sin una clara disposición paralela con espacios entre las fibras (Fig. 2h, i). Los núcleos se presentaron intensamente teñidos y las estriaciones no se distinguieron con claridad.

Notocorda (Fig. 2j-1): La notocorda está compuesta de tejido cordoide vesicular en el cual predominan células llenas de líquido de aspecto vesiculoso; es la estructura parenquimatosa más larga del cuerpo. Se encuentra ventralmente ubicada respecto a la medula espinal situada en la línea media dorsal conjuntamente con la aorta dorsal sobre su línea media ventral, y está rodeada de una capa fibroconjuntiva que forma la cubierta externa. En general la notocorda es turgente y muy distendida, en las larvas saludables se caracteriza por estar en contacto con la musculatura del tronco (Fig. 2j), pero en larvas en mala condición puede mostrar cierto grado de contracción y separación de la musculatura (Fig. 2k, 1).

\section{DesCRIPCIÓN hISTOLÓGICA PARA DIAGNOSIS A CORTO PLAZO}

Tubo digestivo (Fig. 3a-c): El tubo digestivo está compuesto de una porción anterior, una intermedia de menor longitud pero mayor diámetro y una porción distal corta. La mucosa es recta al comienzo de la etapa larval y luego muestra pliegues leves longitudinales en toda su extensión. Los pliegues de la mucosa en las larvas en estadios avanzados de desarrollo son transversales lo que da un aspecto estriado a la vista externa.

El epitelio de la mucosa en la faringe es estratificado y sólo se encuentran células caliciformes. Esta porción mostró una leve variación a lo largo de la ontogenia larval por lo que no se utiliza en los diagnósticos de condición. Es importante mencionar que las larvas de anchoíta no presentan un estómago diferenciado, en sentido morfológico y glandular, hasta llegar a un estado de madurez muy avanzado.

El epitelio de la mucosa de la parte media del intestino presenta conspicuas microvellosidades (Fig. 3a). El citoplasma tiende a ser homogéneo, basófilo y levemente 

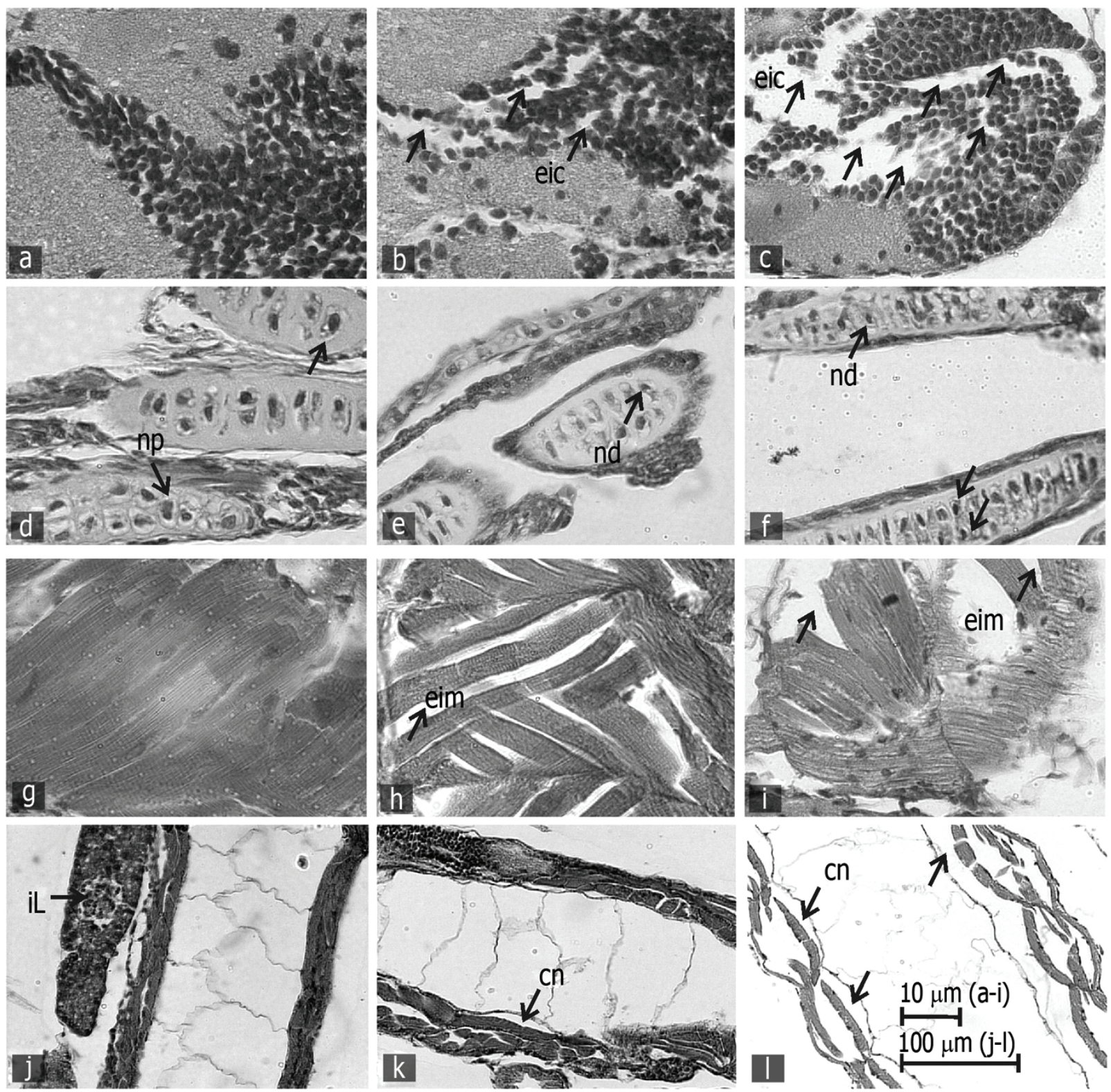

Figura 2. Cortes histológicos de larvas de Engraulis anchoita que caracterizan los diferentes niveles de condición nutricional para los tejidos de largo plazo, (a-c) sistema nervioso, (d-f) cartílago, $(g-i)$ músculo, $(j-l)$ notocorda; izquierda = buena condición, centro = condición intermedia y derecha = mala condición. cn: contracción notocordal, eic: espacio inter-celular, eim: espacio inter-muscular, iL: islote de Langehans, nd: núcleo denso, np: núcleo prominente / Histological sections of Engraulis anchoita larvae that characterize the different levels of nutritional condition for long-term tissue, $(a-c)$ nervous system, $(d-f)$ cartilage, $(g-i)$ muscle, $(j-l)$ notochord; left $=$ healthy condition, middle $=$ intermediate condition and right $=$ poor condition. $\mathrm{cn}$ : notochordal contraction, eic: inter-cellular space, eim: intermuscular space, iL: Langehans islet, nd: dense nucleus, np: prominent nucleus 
granular. Las células se encuentran estrechamente unidas en la superficie lateral y los núcleos están en la porción basal. Las células se deprimen y expanden cuando el lumen es amplio. La porción media mostró una vacuolización y células hipertróficas en algunos especimenes.

Los indicadores típicamente utilizados para diagnosticar la condición nutricional a nivel de la mucosa de la porción media del tubo digestivo son: 1) la textura y la reacción cromática del citoplasma, 2) la prominencia del patrón en mosaico del epitelio o la tendencia a la separación de las células y 3) el aspecto de la banda de microvellosidades. En las larvas saludables se observó un patrón en mosaico de la mucosa prominente, las células se observaron asociadas y con un lumen amplio. Las larvas en inanición presentaron las células de la mucosa intestinal con cierta separación y algo encogidas, atróficas, con formas irregulares y el citoplasma oscuro (Fig. 3b, c).

Las dos grandes glándulas anexas del tracto digestivo (hígado y páncreas) son órganos elongados, compactos, por debajo y parcialmente rodeados por el tubo digestivo. Ambos desembocan por medio de ductos en la porción pilórica del intestino medio con el conducto cístico de la vejiga gaseosa, la cual es una estructura cilíndrica, membranosa en la parte anterior del hígado y el páncreas.

Páncreas (Fig. 3d-f): El páncreas exócrino se ubica a lo largo del lado derecho de la porción anterior del tubo

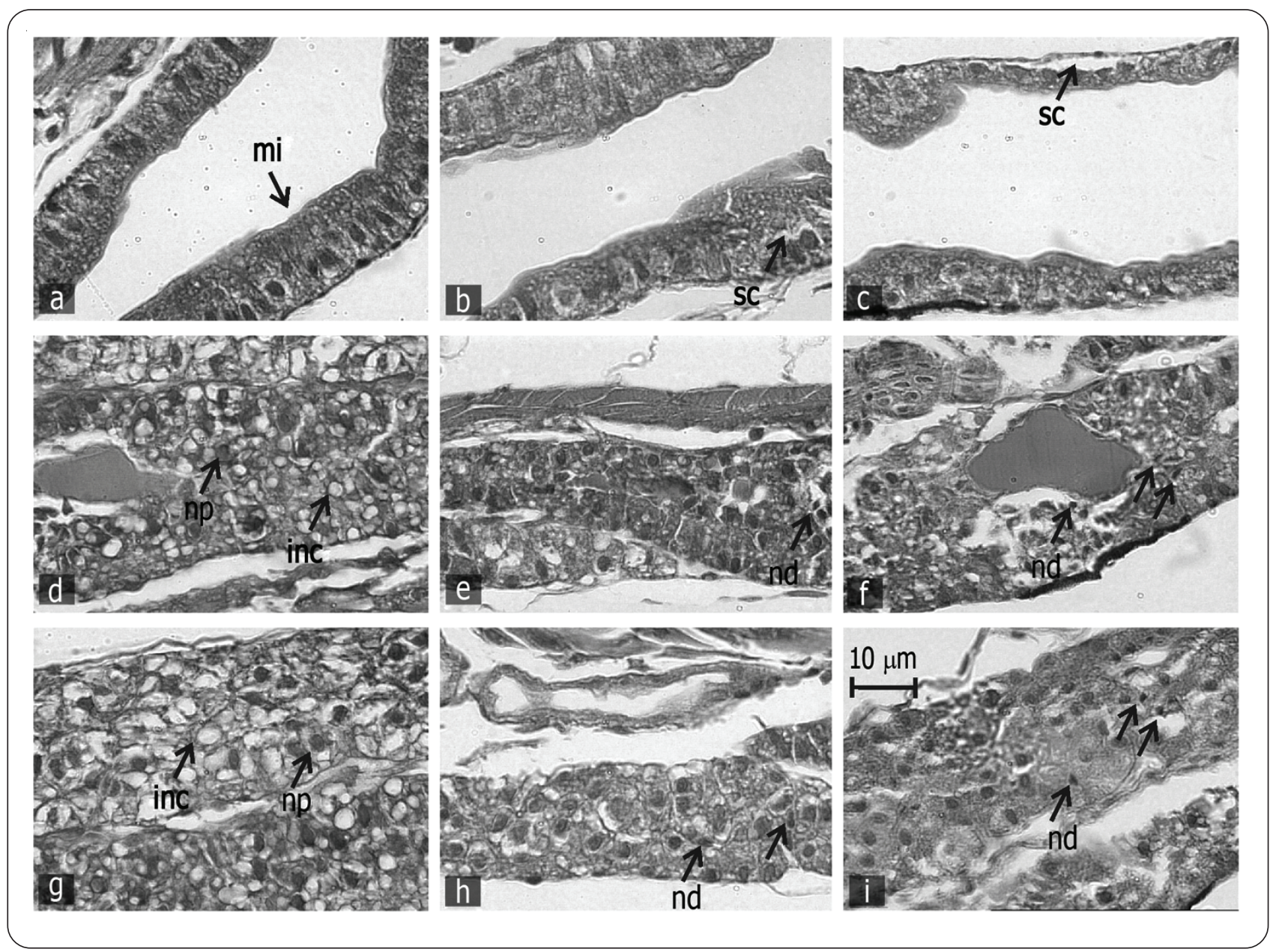

Figura 3. Cortes histológicos de larvas de Engraulis anchoita que caracterizan los diferentes niveles de condición nutricional para los tejidos de corto plazo, (a-c) tubo digestivo, (d-f) páncreas, (g-i) hígado; izquierda = buena condición, centro = condición intermedia y derecha $=$ mala condición. inc: inclusiones, mi: microvellosidades, nd: núcleo denso, np: núcleo prominente, sc: separación celular / Histological sections of Engraulis anchoita larvae that characterize the different levels of nutritional condition for short-term tissue, (a-c) digestive duct, (d-f) pancreas, ( $\mathrm{g}-\mathrm{i})$ liver; left = healthy condition, middle = intermediate condition and right = poor condition. inc: inclusions, mi: microvilli, nd: dens nucleus, np: prominent nucleus, sc: cellular separation

436 Diaz et al.

Descripción micro-anatómica de larvas de Engraulis anchoita 
digestivo, compuesto por acinos de células piramidales rodeados por las ramas terminales del sistema de ductos pancreático. En los estadios tempranos de desarrollo los ductos no son visibles. En las células pancreáticas se observa un núcleo basal basófilo y en la parte apical acumulaciones eosinófilas de cimógeno. El páncreas endócrino presenta un único islote de Langerhans localizado en la masa pancreática, detrás del ducto pancreático (Fig. 2j). En larvas saludables el tejido pancreático se observó compacto, los núcleos celulares prominentes y nucleolos conspicuos; células con neta bipolaridad basófila basal y acidófila en la porción apical (Fig. 3d). Las larvas en mala condición presentaron células pancreáticas pequeñas, disociadas y sus núcleos densos, intensamente teñidos (Fig. 3e, f). El contenido de cimógeno no fue utilizado para caracterizar la condición de los ejemplares.

Hígado (Fig. 3g-i): El hígado como glándula tubulosa compuesta, está estructurado como compacta formación trabecular, cuyas células en la parte basal asientan sobre la red de capilares sinusoides y cuya parte apical delimita los túbulos de los capilares biliares. Los núcleos y el citoplasma de los hepatocitos mostraron gran variabilidad de acuerdo a la condición de los ejemplares. En las larvas saludables, los núcleos fueron evidentes y se observó la presencia del nucleolo, citoplasma hialino en algunos ejemplares, uniforme o con gránulos en otros. Los hepatocitos presentan inclusiones de glucógeno o lípidos, observadas como gránulos o vacuolas respectivamente (Fig. 3g). Debido a que la tinción empleada no permite detectar específicamente estas sustancias, los sitios donde presuntamente estaba el glucógeno se observaron como espacios claros irregulares, dando al citoplasma un aspecto delicadamente alveolar. Por otra parte, las inclusiones lipídicas se observaron como vacuidades redondeadas por la disolución de esta sustancia causada por los solventes utilizados durante el procesamiento histológico. En las larvas que dieron evidencias de mala condición los núcleos fueron picnóticos, oscuros e irregulares, el citoplasma reducido y sin espacios intracelulares (Fig. 3h, i).

\section{ÍNDICE DE CONDICIÓN HISTOLÓGICA}

Los valores de ICH obtenidos para los diferentes ED presentó diferencias significativas empleando todos los tejidos $\left(\mathrm{F}_{(5,189)}=5,39 ; P<0,0001\right)$, los de corto plazo $\left(\mathrm{F}_{(5,}\right.$ $\left.{ }_{189)}=4,45 ; P<0,0001\right)$ y los de largo plazo $\left(\mathrm{F}_{(5,189)}=2,52\right.$; $P<0,05)$. Las comparaciones de los valores ICH entre los ED mediante el test a posteriori, mostraron diferencias significativas entres estados (test Tukey $P<0,005$ ) considerando todos los tejidos (Fig. 4a), los de corto plazo (Fig. 4b) y los de largo plazo (Fig. 4c).

El ICH a largo plazo permitió únicamente encontrar diferencias significativas entre el ED II y el VI, los estadios restantes no difirieron significativamente de estos dos. El ICH a corto plazo permitió encontrar una mayor cantidad de diferencias significativas entre los ED de los ejemplares y el ICH, empleando todos los tejidos, presentó una situación intermedia.

Se destaca que el ICH a corto plazo mostró una reducción significativa en el estadio de desarrollo II, así

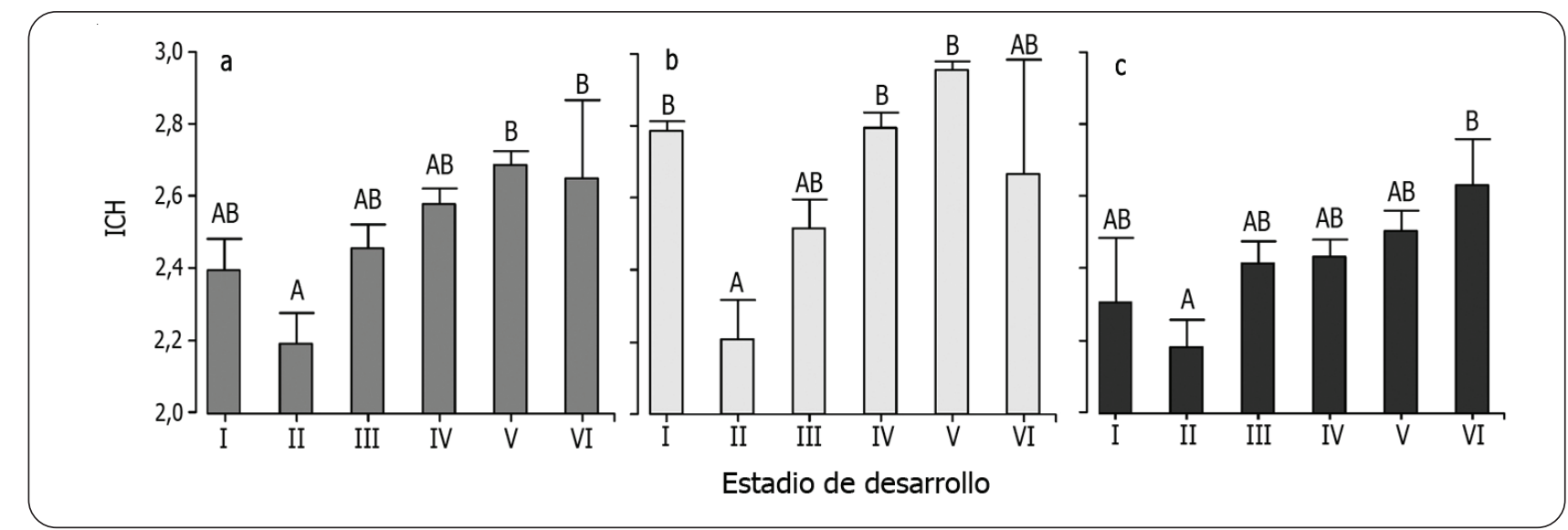

Figura 4. Índice de Condición Histológico (ICH) medio en función del estadio de desarrollo en larvas de Engraulis anchoita. Promedio de todos los tejidos estudiados (a), empleando tejidos de corto plazo (b) y de largo plazo (c). Las letras mayúsculas distintas indican diferencias significativas en las comparaciones de Tukey $(P<0,001)$. Las barras verticales indican el error estándar / Mean values of Histological Condition Index (ICH) for Engraulis anchoita larvae from each developmental stage. Average of all tissues studied (a), employing short term tissues (b) and long term tissues (c). Different letters indicate significant differences $(P<0.001)$ among developmental stages, after ANOVA followed by post-hoc Tukey's (HSD) test. Vertical bars indicate standard error 
como también una pequeña reducción en el estadio VI (Fig. 4b). El ICH a largo plazo solo mostró una reducción durante el estadio de desarrollo II (Fig. 4c).

\section{Discusión}

El efecto de la disponibilidad de alimento sobre la condición nutricional de los individuos depende de la constitución individual de los ejemplares. A medida que las larvas se desarrollan se hacen más resistentes a la inanición debido al incremento en la acumulación de reservas. Si la cantidad de alimento disponible disminuye, las larvas de mayor edad podrían tolerar las condiciones desfavorables durante más tiempo empleando sus reservas de lípidos y glucógeno (Blaxter \& Ehrlich 1974, Powell \& Chester 1985). Por lo tanto, cuando se pretende estudiar la incidencia de la inanición en poblaciones naturales, se debería contemplar la edad o estadio de desarrollo de los ejemplares al momento de realizar comparaciones. El hecho de que las longitudes de los ejemplares de los distintos estadios de desarrollo se solapen indica que la longitud no es un buen indicador del desarrollo o la edad larval. Por otra parte, como consecuencia de la amplia distribución de las áreas de desove de la anchoíta, sus estadios tempranos de desarrollo pueden ser encontrados en zonas con escenarios oceanográficos muy diferentes, y en particular a temperaturas muy diferentes. La temperatura representa uno de los principales factores con influencia sobre el desarrollo larval (Jobling 1995) y por consiguiente la relación longitud-edad de las larvas puede variar significativamente a diferentes latitudes. Resulta evidente que la longitud larval provee información restringida sobre el estadio de desarrollo, que es esencial para estimar la incidencia de la inanición en las distintas fases del incremento somático o empleando material recolectado en zonas con diferentes temperaturas. Debido a que el estudio de crecimiento mediante otolitos en general no es compatible con la histología, las comparaciones deberían hacerse entre ejemplares del mismo estadio de desarrollo y no entre ejemplares de una misma longitud.

El patrón micro-anatómico observado en este estudio fue similar a lo descrito para Engraulis mordax por O'Connell (1981) bajo condiciones controladas de laboratorio. Debido a que los resultados aquí presentados provienen de observaciones realizadas sobre material recolectado en ambiente natural, algunas diferencias cronológicas en el desarrollo podrían esperarse al comparar la micro-anatomía de larvas de E. anchoita bajo condiciones controladas de laboratorio y pueden deberse a diversas fuentes de variabilidad que operan en el océano y suelen ser controladas en estudios realizados bajo condiciones de laboratorio.

Todos los tejidos caracterizados en este trabajo han sido ampliamente utilizados para determinar la condición de larvas de peces tanto en laboratorio como en el medio natural (O'Connell 1976, Theilacker 1978, McFadzen \& Franceschini 1997, Sieg 1998). En particular, el sistema digestivo de las larvas privadas de alimento muestra un deterioro progresivo en los tejidos y en la cito-estructura, aún durante breves periodos de inanición (Gisbert \& Doroshov 2003). En general, el patrón de cambios histológicos observado en teleósteos frente a la inanición incluye: cambios en la organización del hígado, una disminución en el glucógeno y en los lípidos almacenados en los hepatocitos (Margulies 1993, Green \& McCormick 1999, Crespo et al. 2001), una reducción en la altura de los enterocitos (Margulies 1993, Bisbal \& Bengtson 1995, Theilacker \& Porter 1995, Green \& McCormick 1999, Gwak et al. 1999) y una degeneración en la estructura del páncreas exócrino (Yúfera et al. 1993, Gwak et al. 1999, Crespo et al. 2001). Conjuntamente con la reducción en las sustancias de reserva durante la inanición ocurre un incremento en los contenidos de agua (Johnston \& Goldspink 1973, Stirling 1976). Los cambios en el tubo digestivo y en el hígado son rápidos debido a su función como tejidos de reserva. El tejido nervioso parece ser el último en mostrar cambios frente a la inanición (Ehrlich et al. 1976). La movilización de las sustancias de reserva parece ser un proceso controlado en los estadios tempranos de desarrollo, mostrando una conservación de la reserva energética en los órganos más vitales para la supervivencia, las que solo son consumidas o transformadas si las condiciones de inanición son extremas.

Theilacker \& Watanabe (1989) emplearon la altura del epitelio intestinal como un índice de condición nutricional para larvas de Engraulis mordax criadas en laboratorio. Estos autores utilizaron larvas en estadios tempranos de desarrollo con el tubo digestivo recto y larvas con el tubo digestivo plegado. Sieg (1998) observó que durante el proceso de plegamiento de la mucosa intestinal, la altura de los epitelios primero decrece para luego volver a incrementarse. Por consiguiente, la altura del epitelio intestinal como indicador de condición solo puede ser utilizada dentro de un cierto estadio de desarrollo, de otra manera conduciría a errores de interpretación. La altura del epitelio intestinal ha demostrado no ser un buen indicador de la condición nutricional en larvas de $E$. 
anchoita recolectadas in situ (Sieg 1998). Por el contrario la apariencia histológica del hígado y el tubo digestivo son estructuras confiables para clasificar la condición de las larvas de esta especie recolectadas en su ambiente natural (Sieg 1998). En el presente estudio, el ICH a largo plazo permitió encontrar diferencias significativas únicamente entre el ED II y el VI. En cambio el ICH a corto plazo mostró una mayor cantidad de diferencias significativas entre los diferentes estadios de desarrollo. Es decir que estos tejidos parecen más sensibles para detectar diferencias en la condición nutricional de las larvas. Diversos estudios han señalado que estos dos tejidos muestran cambios claros frente a la inanición, ya sea en estudios de laboratorio como en el océano (Sieg 1992, 1995, Ferron \& Leggett 1994).

La reducción observada en el ICH durante el estadio de desarrollo II indicaría que luego de la reabsorción del vitelo, que ocurre al finalizar el estadio de desarrollo I, la condición fisiológica de las larvas se reduciría y se hizo evidente empleando tanto los tejidos de corto como los de largo plazo. En esta etapa, llamada también período crítico (Hjort 1914) las larvas se enfrentan a grandes requerimientos energéticos y si no logran acceder con relativa rapidez al alimento apropiado, corren riesgo de sufrir inanición y en consecuencia retardar su crecimiento o morir (May 1974, Theilacker 1978). Como resultado, si la fuente de alimento es escasa o la captura de presas no es adecuada, las posibilidades de supervivencia se reducen significativamente. Por este motivo, el período crítico larval es considerado una de las mayores causas de mortalidad en muchas especies de peces marinos (Hunter 1976)

Por otra parte la reducción en el ICH a corto plazo observada en el estadio VI indicaría que la metamorfosis hacia la etapa juvenil también representaría en esta especie un período crítico de grandes requerimientos energéticos para las larvas, en el cual la probabilidad de supervivencia se reduciría. Ciechomski et al. (1986) observaron un patrón similar en el comportamiento del factor de condición en larvas de E. anchoita y registraron una reducción importante durante el periodo de reabsorción del vitelo, una mejora posterior y una nueva reducción hacia el final de la etapa larval. Este patrón fue relacionado con cambios en el desarrollo de los ejemplares. Estudios futuros empleando técnicas morfométricas e histológicas simultáneamente permitirían verificar la correspondencia entre los cambios en la morfometría y las alteraciones histológicas durante estas dos etapas del periodo larval.
Los ejemplares del ED I usualmente no son considerados para los estudios de condición nutricional ya que, debido a su alimentación endógena, su condición fisiológica sería básicamente reflejo de las influencias maternas, más que de su condición nutricional exógena vinculada al ambiente. Sin embargo en este estudio han sido incluidos con fines comparativos para estimar la condición inicial o maternal de las larvas.

Cabe aclarar que de acuerdo a observaciones previas realizadas por Diaz et al. (2011), las larvas de anchoíta presentaron valores medios de ICH entre 2 y 3 indicando una condición moderada a saludable. Estos autores empleando técnicas morfométricas, histológicas y la relación ARN/ADN han observado que las larvas de anchoíta de la población bonaerense de la especie se encuentran en condición saludable al menos durante el período de estudio. En este sentido se destaca la sensibilidad del ICH a corto plazo que aún frente a una condición saludable de las larvas permitió distinguir aquellas con una pequeña reducción en su condición.

Dentro de la gran variedad de posibilidades para el estudio de la condición nutricional de larvas de peces, las técnicas histológicas han sido caracterizadas como de gran potencial. A pesar que requieren una inversión de tiempo importante, siguen siendo utilizadas ampliamente. El estudio histológico puede ser focalizado en los tejidos que mejor representen las variaciones para la especie estudiada, el estadio de desarrollo y la escala temporal de interés, en función del objetivo de estudio. Uno de los problemas es la difícil objetivación de los métodos de valoración de las características de los tejidos o células, ya que las medidas suelen basarse en apreciaciones cualitativas o semi-cualitativas ( $\mathrm{O}^{\prime}$ Connell 1980). Si bien las apreciaciones cualitativas son más sencillas de obtener, plantean el problema de la variabilidad introducida por la experiencia del observador (Ferron \& Leggett 1994).

Recientemente, los estudios de análisis de imágenes digitales son más difundidos, permitiendo realizar una cuantificación del diagnóstico histológico para su posterior análisis estadístico. Sin embargo, Catalán (2003) ha comparado mediciones cualitativas y cuantitativas realizadas sobre individuos sometidos a tratamientos de alimentación y ha observado que los métodos cualitativos permiten la correcta clasificación de los individuos en la mayoría de los casos y para todos los estadios de desarrollo. Esta situación ha sido atribuida a que los índices cualitativos consideran una mayor cantidad de características tisulares y celulares que los índices cuantitativos. 
Debido a la gran sensibilidad que muestran frente a la inanición en estudios de laboratorio y de campo, el tubo digestivo y glándulas anexas son usualmente los tejidos empleados para realizar los diagnósticos histológicos de larvas de peces (Peña \& Dumas 2005, Catalán et al. 2006, Chen et al. 2007, Díaz et al. 2011). Sin embargo, como consecuencia de la respuesta diferencial frente a la inanición, los distintos tejidos reflejan cambios a corto o largo plazo, por lo que el tipo de índice que se utilice depende de la escala temporal con la que se desee trabajar. Para la elección del índice más apropiado a utilizar, también debe considerarse la especie que se estudia, el estadio de desarrollo en que se encuentran los especímenes y la pregunta que se quiere responder (Suthers 1998).

Por lo tanto, el análisis histológico del sistema digestivo de las larvas de la población bonaerense de anchoíta (en particular del hígado y el tubo digestivo) representaría un indicador sensible de su condición nutricional y podría ser empleado para estimar la incidencia de la inanición en la mortalidad larval a lo largo de su desarrollo ontogénico.

\section{Agradecimientos}

Los autores expresan su gratitud a Marta Estrada por su gentil colaboración en los procesamientos histológicos; a las autoridades del INIDEP y al personal embarcado. Finalmente, se agradece a los revisores anónimos del manuscrito por sus valiosos comentarios y aportes. Contribución INIDEP n ${ }^{\circ} 1700$.

\section{LITERATURA CITADA}

Bisbal G \& DA Bengtson. 1995. Descriptions of the starving condition in summer flounder, Paralichthys dentatus, early life history stages. Fishery Bulletin 93: 217-230.

Blaxter JHS \& KF Ehrlich. 1974. Changes in behaviour during starvation of herring and plaice larvae. En: Blaxter JHS (ed). The early life history of fish, pp. 575-588. SpringerVerlag, New York.

Brandhorst W, JP Castello, MB Cousseau \& DA Capezzani. 1974. Evaluación de los recursos de anchoíta (Engraulis anchoita) frente a Argentina y Uruguay. VIII. Desove, crecimiento, mortalidad y estructura de la población. Physis A 33(86): 37-58.

Catalán IA. 2003. Condition indices and their relationship with environmental factors in fish larvae. Doctoral Thesis, Universitat de Barcelona, Barcelona, 265 pp.

Catalán IA, MP Olivar, I Palomera \& E Berdalet. 2006. Link between environmental anomalies, growth and condition of pilchard Sardina pilchardus (Walbaum) larvae in the NW Mediterranean. Marine Ecology Progress Series 307: 219-231.
Chen NB, JG Qin, JF Carragher, SM Clarke, MS Kumar \& WG Hutchinson. 2007. Deleterious effects of food restrictions in yellowtail kingfish Seriola lalandi during early development. Aquaculture 271(1-4): 326-335.

Ciechomski JD. 1965. Observaciones sobre la reproducción, desarrollo larval embrionario y larval de la anchoíta argentina (Engraulis anchoita). Boletín del Instituto de Biología Marina 9: 1-29.

Ciechomski JD \& RP Sánchez. 1988. Análisis comparativo de las estimaciones de biomasa de la anchoita (Engraulis anchoita) en el Atlántico Sudoccidental en diferentes años y con distintas metodologías. Publicación de la Comisión Técnica Mixta del Frente Marítimo 4: 117-131.

Ciechomski JD, RP Sánchez, G Alespeiti \& H Regidor. 1986. Estudio sobre el crecimiento en peso y factor de condición en larvas de anchoíta, Engraulis anchoita Hubbs \& Marini. Variaciones regionales, estacionales y anuales. Revista de Investigación y Desarrollo Pesquero 5: 183-193.

Crespo S, M Marín de Mateo, CA Santamaría, R Sala, A Grau \& E Pastor. 2001. Histopathological observations during larval rearing of common dentex Dentex dentex L. (Sparidae). Aquaculture 192: 121-132.

Diaz MV, M Pájaro, MP Olivar, P Martos \& GJ Macchi. 2011. Nutritional condition of Argentine anchovy Engraulis anchoita larvae in connection with nursery ground properties. Fisheries Research 109: 330-341.

Ehrlich KF, JHS Blaxter \& R Pemberton. 1976. Morphological and histological changes during the growth and starvation of herring and plaice larvae. Marine Biology 35: 105-118.

Ferron A \& WC Leggett. 1994. An appraisal of condition measures for marine fish larvae. Advances in Marine Biology 30: 217-303.

Fuiman LA. 2002. Special considerations of fish eggs and larvae. En: Fuiman LA \& RG Werner (eds). Fishery science: the unique contributions of early life stages, pp. 1-32. Blackwell Publishing Professional, New York.

Gisbert E \& SI Doroshov. 2003. Histology of the developing digestive system and the effect of food deprivation in larval green sturgeon (Acipenser medirostris). Aquatic Living Resources 16: 77-89.

Green BS \& MI McCormick. 1999. Influence of larval feeding history on the body condition of Amphiprion melanopus. Journal of Fish Biology 55: 1273-1289.

Gwak WS, T Seikai \& M Tanaka. 1999. Evaluation of starvation status of laboratory reared Japanese flounder Paralichthys olivaceus larvae and juveniles based on morphological and histological characteristics. Fisheries Science 65: 339-346.

Hjort J. 1914. Fluctuations in the great fisheries of northern Europe viewed in the light of biological researches. Rapports et Procès-verbaux des Réunions, Conseil International pour l'Exploration de la Mer 20: 1-128. 
Hunter JR. 1976. Culture and growth of northern anchovy (Engraulis mordax) larvae. Fishery Bulletin 74(1): 81-88.

Jobling M. 1995. Development of eggs and larvae. En: Jobling M (ed). Environmental biology of fishes, pp. 357-390. Chapman \& Hall, London.

Johnston IA \& G Goldspink. 1973. Some effects of prolonged starvation on metabolism of the red and white myotomal muscles of the plaice Pleuronectes platessa. Marine Biology 19: 348-353.

Margulies D. 1993. Assessment of the nutritional condition of larval and early juvenile tuna and Spanish mackerel (Pisces: Scombridae) in the Panamá Bight. Marine Biology 115: 317-330.

May RC. 1974. Larval mortality in marine fishes and the critical period concept. En: Blaxter JHS (ed). The early life history of fish, pp. 3-19. Springer-Verlag, New York.

McFadzen IRB \& G Franceschini. 1997. The nutritional condition of larvae of anchovy (Engraulis encrasicolus L.) in the outflow of the River Po (Northern Adriatic). Acta Adriatica 38(1): 49-64.

McFadzen IRB, DM Lowe \& SH Coombs. 1994. Histological changes in starved turbot larvae (Scophthalmus maximus) quantified by digital image analysis. Journal of Fish Biology 44: $255-262$.

O'Connell CP. 1976. Histological criteria for diagnosing the starving condition in early post yolk sac larvae of the northern anchovy, Engraulis mordax Girard. Journal of Experimental Marine Biology and Ecology 25: 285-312.

O'Connell CP. 1980. Percentage of starving northern anchovy, Engraulis mordax, larvae in the sea as estimated by histological methods. Fishery Bulletin 78(2): 475-489.

O'Connell CP. 1981. Development of organ systems in the northern anchovy, Engraulis mordax, and other teleosts. American Zoologist 21: 429-446.

Oozeki Y, T Ishii \& R Hirano. 1989. Histological study of the effects of starvation on reared and wild-caught larval stone flounder, Kareius bicoloratus. Marine Biology 100: 269-275.

Peña R \& S Dumas. 2005. Effect of delayed first feeding on development and feeding ability of Paralabrax maculatofasciatus larvae. Journal of Fish Biology 67: 640651.

Phonlor G. 1984. Morfologia e biologia de larvas de Engraulis Anchoita (Hubbs \&Marini), (Osteichthyes, Engraulidae). Atlântica 7: 85-98.

Powell AB \& AJ Chester. 1985. Morphometric indices of nutritional condition and sensitivity to starvation of spot larvae. Transactions of the American Fisheries Society 114: 338-347.
Sánchez RP. 1995. Patrones de distribución espacio-temporal de los estadios embrionarios y larvales de la anchoíta (Engraulis anchoita Hubbs \& Marini) a micro y macro escala, su relación con la supervivencia y el reclutamiento. Tesis Doctoral, Universidad Nacional de Buenos Aires, Buenos Aires, 672 pp.

Sieg A. 1992. Histological study of organogenesis in the young stages of the mesopelagic fish Vinciguerria (Photichthyidae, Pisces). Bulletin of Marine Science 50(1): 97-107.

Sieg A. 1995. Histologische Kriterien zur Erfassung unterschiedlicher Ernährungszustände bei frühen Lebensstadien der Südwestatlantischen Sardelle, Engraulis anchoita Hubbs und Marini 1935, und das Auftreten verschieden ernährter Larven dieser Art in hydrographisch abweichenden Regionen ihres Rekrutierungsgebietes. Doctoral Thesis, Berichte aus dem Zentrum für Meeresund Klimaforschung der Universität Hamburg, Hamburg, 159 pp.

Sieg A. 1998. A study on the histological classification of the in situ-nutritional condition of larval South-west Atlantic anchovy, Engraulis anchoita Hubbs and Marini, 1935. Archive of Fishery and Marine Research 46(1): 19-36.

Stirling HP. 1976. Effects of experimental feeding and starvation on the proximate composition of the European bass Dicentrarchus labrax. Marine Biology 34: 85-91.

Suthers IM. 1998. Bigger? Fatter? Or is faster growth better? Considerations on condition in larval and juvenile coralreef fish. Australian Journal of Ecology 23: 265-273.

Theilacker GH. 1978. Effect of starvation on the histological and morphological characteristics of jack mackerel, Trachurus symmetricus, larvae. Fishery Bulletin 76: 403414.

Theilacker GH \& SM Porter. 1995. Condition of larval walleye pollock Theragra chalcogramma, in the Western Gulf of Alaska, assessed with histological and shrinkage indices. Fishery Bulletin 93: 333-344.

Theilacker GH \& Y Watanabe. 1989. Midgut cell heigth defines nutritional status of laboratory-raised larval northern anchovy, Engraulis mordax. Fishery Bulletin 87: 1989-1998.

Yúfera M, E Pascual, A Polo \& MC Sarasquete. 1993. Effect of starvation on the feeding ability of gilthead seabream (Sparus aurata L.) larvae at first feeding. Journal of Experimental Marine Biology and Ecology 169: 259-272.

Watanabe Y. 1985. Histological changes in the liver and intestine of freshwater goby larvae during short-term starvation. Bulletin of the Japanese Society for the Science of Fish 51: 707-710.

Recibido el 26 de enero de 2011 y aceptado el 14 de noviembre de 2011 